\title{
Tempobezeichnungen von Julius Stockhausen für Die schöne Müllerin: Ein Quellenfund
}

\author{
CHRISTIAN LEHMANN, MÜNCHEN
}

Am 4. Mai 1856 sang der Bariton Julius Stockhausen im Wiener Musikvereinssaal zum ersten Mal den gesamten Liederzyklus Die schöne Müllerin von Franz Schubert in einem öffentlichen Konzert. Mit dieser Aufführung schrieb Stockhausen Geschichte - war es doch zu seiner Zeit übliche Praxis, für ein kammermusikalisches Konzertprogramm einzelne Stücke unterschiedlicher Gattungen zusammenzustellen. Das „Experiment"1 des Sängers legte den Grundstein für eine höhere Wertschätzung des Kunstliedes als konzertwürdige Gattung und trug wesentlich zu der Auffassung bei, einen Liederzyklus als zusammenhängendes Werk zu sehen. Stockhausen wiederholte die Gesamtaufführung der Schönen Müllerin zahlreiche Male, darunter in Hamburg mit Johannes Brahms am Klavier.

Der 1826 als Kind deutscher Eltern in Paris geborene Julius Stockhausen, zweisprachig aufgewachsen und am Pariser Konservatorium bei Manuel Garcia ausgebildet, erlangte auch als Dirigent Geltung. 1862 wurde ihm die Leitung der Philharmonischen Konzerte in Hamburg übertragen - eine Position, auf die der mit Stockhausen befreundete Johannes Brahms gehofft hatte. 1874 bis 1878 stand Stockhausen dem Stern'schen Gesangsverein zu Berlin vor.

1878 wurde er als Gesangspädagoge an das Hoch'sche Konservatorium in Frankfurt am Main berufen und setzte später seine Unterrichtstätigkeit in einer eigenen Frankfurter Gesangsschule fort. Wenn auch die Urteile über Stockhausens Gesangstechnik und die Gültigkeit seiner Lehre auseinandergehen, so ist doch seine historische Bedeutung als Interpret in seiner Zeit und wichtige Musikerpersönlichkeit im engen Umfeld von Johannes Brahms und dem Ehepaar Amalie und Joseph Joachim unbestritten, vor allem aber seine gesangspädagogische Wirkungsgeschichte. Davon zeugen die Verbreitung seiner theoretischen Schriften sowie die zahlreichen prominenten Sängerpersönlichkeiten, die durch seine Schule gingen und über „Enkelschüler" wie z. B. Franziska Martienssen-Lohmann noch die heutige Generation prägen.

Schuberts Schöne Müllerin spielte für Julius Stockhausen Zeit seines Lebens eine herausragende Rolle; er brachte den Zyklus auch nach Ende seiner aktiven Sängerlaufbahn in Schülerkonzerten zur Aufführung.

1 Eduard Hanslick, Geschichte des Concertwesens in Wien, Zweiter Theil, Wien 1870, S. 214. 


\section{Das Fundstück}

Im Nachlass des deutschen Baritons Gerhard Hüsch (1901-1984) befand sich ein mit Füllfederhalter beschriebenes Blatt im Format DIN A4, das folgende Überschrift trägt: „Tempobezeichnungen von Julius Stockhausen, Frühjahr 1876". Darunter steht die Werkbezeichnung "Die schöne Müllerin", dann folgen tabellarisch Metronomzahlen für jede einzelne Liednummer mit Ausnahme der Nummer 13 sowie folgende Anmerkungen: „Die Schrift (Bleistift) ist im Laufe der Jahrzehnte verblasst. Um mich nicht an dem Andenken Stockhausen's zu versündigen, habe ich alle Bezeichnungen mit meinem Metronom nachgeprüft. - Nr. 6 I und Nr. 10 haben mich allerdings einigermassen überrascht." Zu Lied Nr. 13 („Mit dem grünen Lautenbande”) „fehlen die Zahlen, trotz anderer Anmerkungen”. Dies wird mit einer Fußnote erläutert: „Max Friedlaender, bei dem ich etliche Müllerlieder einst studierte, und der im Grossen und im Kleinen auf seinen Meister Stockhausen schwor, nahm das Tempo $\delta=104$ etwa. - Die in meinem Besitz befindliche Stockhausen-Müllerlieder-Ausgabe stammt nicht von Max Friedlaender."

Verfasserin bzw. Kopistin des Dokuments ist die Berliner Gesangsprofessorin Clara Klatte (1867-1945). Sie signierte das Blatt mit ihren Initialen und dem Datum „18.9.36". Wahrscheinlich übereignete sie das Dokument Gerhard Hüsch, der Mitte der 1930er Jahre in Berlin lebte und in dieser Zeit einer der prominentesten deutschen Liedinterpreten war. Nach dem Krieg vor allem als Gesangspädagoge gefragt, verwendete Hüsch das Papier offensichtlich als Arbeitsblatt. Davon zeugt nicht nur der deutlich gebrauchte Zustand des Dokuments. Hüsch erweiterte Clara Klattes Tabelle und die Rückseite des Blattes auch mit Tempoangaben des Stockhausen-Schülers Johannes Messchaert, die dieser in den 1920er Jahren in einer SchubertAusgabe publiziert hatte.

\section{Eine glaubwürdige Quelle?}

Bisher bekannte Quellen liefern keine Hinweise darauf, dass Julius Stockhausen Tempoangaben für Schubertlieder oder andere Werke vorgenommen hat. Von ihm vorgeschlagene Metronomzahlen wären also ein bislang unbekanntes Novum. Wenn die Angaben authentisch sind, sind sie von hohem musikwissenschaftlichen Interesse, da die auf dem Dokument angegebenen Tempi teilweise überraschen. Daher gilt es zuerst, die Frage zu stellen: Wie glaubwürdig ist die Quelle? Was spricht dafür oder dagegen, dass die darauf notierten Angaben wirklich von Julius Stockhausen stammen? Um dieser Frage nachzugehen, habe ich untersucht, ob Verbindungen zwischen Julius Stockhausen und der Verfasserin des Manuskripts - Clara Klatte - nachweisbar sind. 


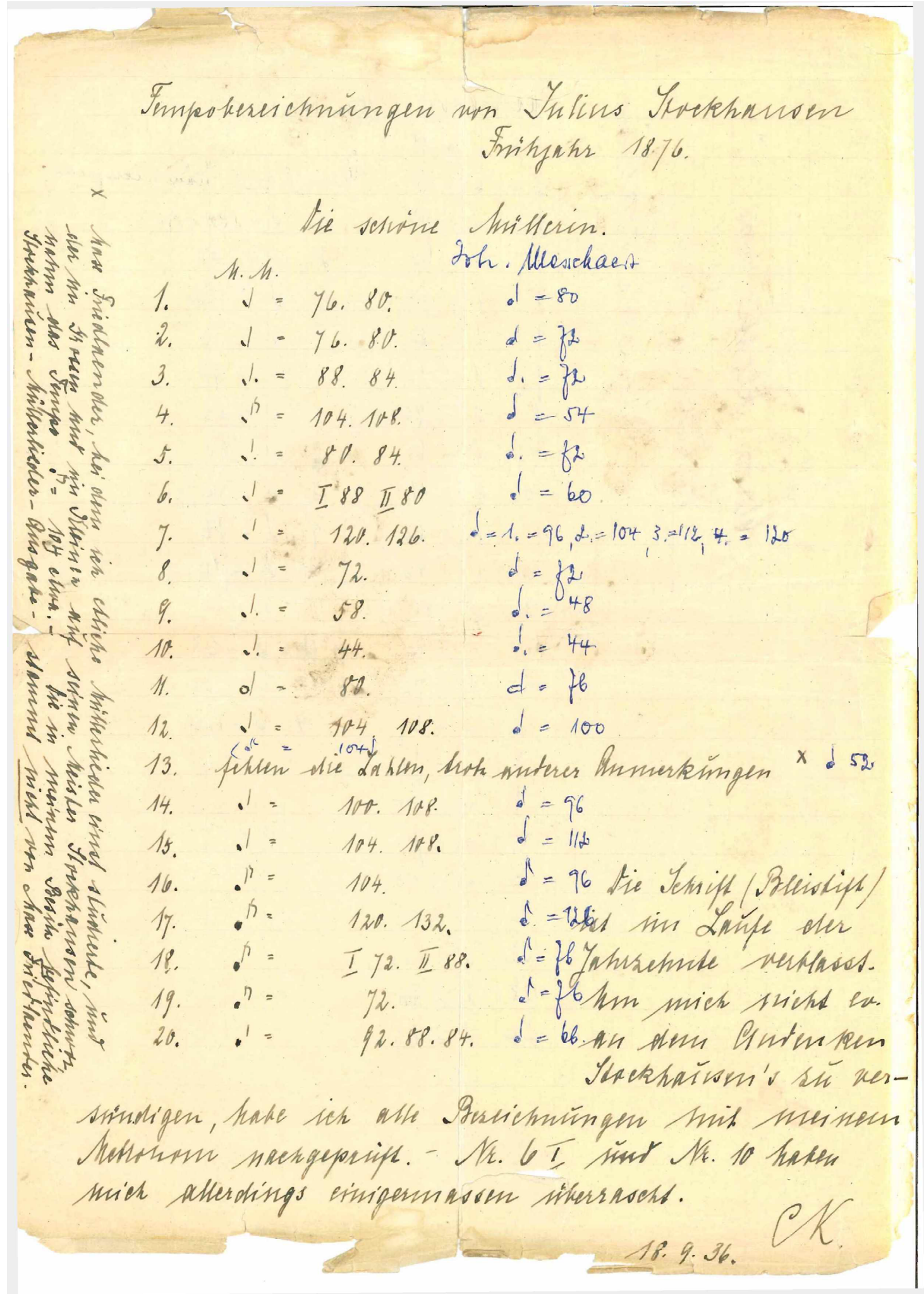

Abbildung 1: Scan der Vorderseite des Manuskripts, „Tempobezeichnungen von Julius Stockhausen, Frühjahr 1876”. Urheberrecht 1.0, <https://rightsstatements.org/page/InC/1.0/?language=de $>$. 
Clara Klatte, geboren 1867 als Baronesse Senfft von Pilsach, ${ }^{2}$ unterrichtete in den 1920er Jahren Gesang am Stern'schen Konservatorium in Berlin. Ihr Ehemann Wilhelm Klatte war am selben Institut Professor für Musiktheorie. Ein Vermerk auf dem fraglichen Dokument, "Clara Klatte war Schülerin von Amalie Joachim", wird durch eine weitere Quelle bestätigt. ${ }^{3}$ Amalie Joachim und ihr Mann, der Geiger Joseph Joachim, standen wiederum in lebenslanger freundschaftlicher Verbindung mit Julius Stockhausen. Die Joachims lebten seit 1868 in Berlin, wo Joseph Joachim der Akademie der Tonkunst vorstand. Clara Klatte datiert die "Tempobezeichnungen" auf das Jahr 1876. Zu dieser Zeit leitete Stockhausen in Berlin den Stern'schen Gesangsverein. Denkbar ist die Konstellation, dass Amalie Joachim Stockhausens Notizen erhielt und diese wiederum ihrer Schülerin Clara überließ - zumal auch Amalie Joachim selbst die Schöne Müllerin aufgeführt hat.

\section{Arnold Senfft von Pilsach}

Doch es gibt noch eine zweite, deutlich kürzere Verbindungslinie: 1834 wurde in Gramenz in Pommern Arnold Senfft von Pilsach als Spross einer Landjunkerfamilie geboren. Der junge Baron mit musischen Neigungen studierte Jurisprudenz und promovierte in Halle an der Saale zum Doktor der Rechte. Hier lernte Arnold von Senfft den Direktor der Halleschen Singakademie und Liederkomponisten Robert Franz kennen. Franz wurde sein musikalischer Lehrer - und Baron von Senfft, ein ambitionierter Dilettant im besten Wortsinne, förderte den Komponisten als Mäzen.

In den 1860er Jahren nahm Arnold von Senfft in Berlin eine leitende Stellung bei einer Versicherung an. Als der berühmte Sänger Julius Stockhausen 1874 nach Berlin kam, nahm Senfft Gesangsunterricht bei ihm und wurde nach dem Urteil zeitgenössischer Kritiken Stockhausens „bedeutendster Schüler" ${ }^{4}$ Schon Mitte der 1870er Jahre trat Senfft immer öfter als Sänger der Lieder von Robert Franz und Balladen von Carl Loewe in Erscheinung, aber auch als Oratoriensänger mit dem Stern'schen Gesangverein unter Stockhausens Leitung.

Arnold Senfft von Pilsach aber war der Vater von Clara Klatte, der Verfasserin des Blattes mit den Metronomangaben. Es ist also nicht abwegig anzunehmen, dass Senfft von seinem Lehrer und Mentor Stockhausen die genannte "Müllerlieder-Ausgabe" mit handschriftlichen Anmerkungen erhielt, die nach seinem Tod in den Besitz seiner Tochter Clara kam.

Arnold Senfft von Pilsach scheint bei seiner Herangehensweise an die Musik ein gewisses Bedürfnis nach messbarer Exaktheit an den Tag gelegt zu haben. Das deutet kein Geringerer

2 Genealogisches Handbuch freiherrliche Häuser, Teil A, Bd. 12, hrsg. von Deutsches Adelsarchiv (= Genealogisches Handbuch des Adels, Bd. 74), Limburg/Lahn 1980.

3 Olga Samaroff Stokowski, An American Musician's Story, New York 1939.

4 Max Runze, "Senfft von Pilsach, Arnold Freiherr", in: Allgemeine Deutsche Biographie, Bd. 34 (1892), S. 23-26, <https://www.deutsche-biographie.de/pnd117473154.html\#adbcontent> (14.04.2020). 
als Theodor Fontane an, der mit der Familie Stockhausen befreundet war und auch Senfft von Pilsach kennenlernte. Fontane berichtet Stockhausen im Dezember 1878:

„Denken Sie sich, Sonntag vor acht Tagen kam er zu mir, um mich zu fragen, ob er mir den Sonntag drauf den ,Archibald Douglas' vorsingen dürfe. Da Sie Senfft besser kennen als ich, so wissen Sie, daß diese Anfrage eine Anmeldung war. [...] Und nun ging es los. [...] Stimmgabel 'raus (mit der Senfft beständig operiert wie andere mit dem Lorgnon), und ,Ich hab es getragen sieben Jahr' brauste durch meine sieben Fuß hohen Hallen. ${ }^{15}$

Noch aufschlussreicher ist in diesem Zusammenhang der Briefwechsel zwischen Arnold Senfft von Pilsach und Robert Franz. Senfft und Franz erörtern mehrmals die Frage, ob Lieder "metrisch oder rhythmisch" zu singen sind. Franz plädiert für einen rhythmisch freieren Vortrag. Singen nach Mälzels Metronom könne "steif und hölzern" klingen. Arnold von Senfft aber bittet den Komponisten Franz, der ihm Lieder zur Aufführung zusendet, wiederholt um Metronomzahlen, teilweise mit auffälligem Nachdruck. Zitat eines Briefes vom 16. November 1877:

„Darf ich Sie bitten, mir metronomisch das Tempo obiger Lieder anzugeben! Wenn Sie morgen schreiben, erhalte ich es Sonntag früh, also noch rechtzeitig.

Vorher möchte ich bitten, Ihr Metronom zu prüfen. Wenn Sie es auf 60 stellen, so müssen 60 Schläge eine Minute ergeben. Das können Sie am besten mit einer Sekunden-Uhr [...], sonst aber auch mit einer gewöhnlichen Uhr bewerkstelligen.

Mit herzlichem Gruß

Ihr ergebenster

Arnold von Senfft."

Es ist also denkbar, dass Arnold Senfft von Pilsach auch seinen Gesangslehrer Stockhausen um Metronomangaben zu Schubert-Liedern bat, die Senfft wahrscheinlich bei ihm studierte. Stockhausen könnte die Zahlen dann eigens für Senfft eingetragen haben. Die von Clara Klatte angegebene Datierung "Frühjahr 1876" fällt in die Zeit, als Senfft sich als Konzertsänger in Berlin etablierte und Metronomangaben offenbar als wichtige Hilfe erachtete. Stockhausen selbst hatte seinen Zenit als aktiver Konzertsänger damals bereits überschritten.

Dass Senffts Tochter Clara Jahrzehnte später als pensionierte Gesangsprofessorin die ererbten Daten ausgerechnet an Gerhard Hüsch weitergab, ist wahrscheinlich kein Zufall. Hüsch hatte zur Zeit der Datierung des Blattes 1936 soeben zusammen mit dem Pianisten Hanns-Udo Müller als erstes Liedduo eine Gesamtaufnahme der Schönen Müllerin auf Schellack vorgelegt - 80 Jahre nach der ersten Gesamtaufführung des Zyklus im Konzertsaal durch Julius Stockhausen. Clara Klatte starb am 15. Februar 1945 in dem kleinen Ort Groß-Gandern in Brandenburg (heute polnisch). Über den Verbleib der "Stockhausen-Müllerlieder-Ausgabe“ aus ihrem Besitz ist nichts bekannt.

5 Julia Wirth, Julius Stockhausen. Der Sänger des deutschen Liedes, Frankfurt a.M. 1927, S. 451.

6 Robert Franz und Wolfgang Golther, Robert Franz und Arnold Freiherr Senfft von Pilsach: Ein Briefwechsel 18611888, Berlin 1907, S. 277. 
Zusammenfassend lässt sich sagen: Vor allem die Bezüge zu Clara Klattes Vater Arnold Senfft von Pilsach lassen es plausibel erscheinen, dass Klattes Manuskript tatsächlich auf Angaben von Julius Stockhausen basiert.

\section{Überraschende Tempi}

Um die angegebenen Zahlen adhoc einschätzen zu können, habe ich zum Vergleich die ungefähren Metronomwerte der Müllerlieder in der sehr bekannten Schallplatteneinspielung mit Dietrich Fischer-Dieskau und Gerald Moore von 1971 in einer Tabelle den Stockhausen-Werten gegenübergestellt. ${ }^{7}$

\begin{tabular}{|c|c|c|c|c|c|c|}
\hline Nr. & Titel & Schuberts Tempobezeichnung & Takt & $\begin{array}{l}\text { Einheit } \\
\text { M.M. }\end{array}$ & $\begin{array}{c}\text { Stockhausen } \\
1876\end{array}$ & $\begin{array}{l}\text { Fi-Di / Moore } \\
1971 \text { (approx.) }\end{array}$ \\
\hline 1 & Das Wandern & Mäßig geschwind & $2 / 4$ & $\mathrm{~V}$ & 76.80. & 88 \\
\hline 2 & Wohin? & Mäßig & $2 / 4$ & V & 76. 80 . & 72 \\
\hline 3 & Halt! & Nicht zu geschwind & $6 / 8$ & $\mathrm{~V}$. & 88.84. & 88 \\
\hline 4 & Danksagung an den Bach & Etwas langsam & $2 / 4$ & A & 104. 108. & 76 \\
\hline 5 & Am Feierabend & Ziemlich geschwind & $6 / 8$ & $\mathrm{~V}$. & 80.84. & 86 \\
\hline 6 & Der Neugierige & Langsam / Sehr langsam & $2 / 4$ & $\mathrm{~V}$ & | $88 \quad|| 80$ & | $42 \quad|| 36$ \\
\hline 7 & Ungeduld & Etwas geschwind & $3 / 4$ & $\mathrm{~V}$ & 120.126 & 118 \\
\hline 8 & Morgengruß & Mäßig & $3 / 4$ & V & 72 & 66 \\
\hline 9 & Des Müllers Blumen & Mäßig & $6 / 8$ & $\mathrm{~V}$. & 58 & 56 \\
\hline 10 & Tränenregen & Ziemlich langsam & $6 / 8$ & V. & 44 & 40 \\
\hline 11 & Mein! & Mäßig geschwind & $4 / 4$ & $\mathrm{H}$ & 80 & 92 \\
\hline 12 & Pause & Ziemlich geschwind & $4 / 4$ & $\mathrm{~V}$ & 104. 108. & 90 \\
\hline 13 & Mit dem grünen Lautenbande & Mäßig & $2 / 4$ & A & (Friedländer: 104) & 108 \\
\hline 14 & Der Jäger & Geschwind & $6 / 8$ & $\mathrm{~V}$. & 100. 108 & 102 \\
\hline 15 & Eifersucht und Stolz & Geschwind & $2 / 4$ & V & 104. 108 & 128 \\
\hline 16 & Die liebe Farbe & Etwas langsam & $2 / 4$ & A & 104 & 80 \\
\hline 17 & Die böse Farbe & Ziemlich geschwind & $2 / 4$ & A & 120.132. & 140 \\
\hline 18 & Trockne Blumen & Ziemlich langsam & $2 / 4$ & $A$ & | $72 \quad 1 \mid 88$ & $166 \quad 1172$ \\
\hline 19 & Der Müller und der Bach & Mäßig & $3 / 8$ & A & 72 & $76-84$ \\
\hline 20 & Des Baches Wiegenlied & Mäßig & $2 / 2$ & $\mathrm{~V}$ & 92.88 .84 & 66 \\
\hline
\end{tabular}

Tabelle 1: "Tempoangaben von Julius Stockhausen 1876", aufgeschrieben von Clara Klatte, im Vergleich zu den Tempi der Aufnahme mit Dietrich Fischer-Dieskau und Gerald Moore 1971

Blau = Stockhausen-Tempi langsamer als bei Fischer-Dieskau/Moore

Rot $=$ Stockhausen-Tempi deutlich schneller als bei Fischer-Dieskau/Moore

$A=$ Achtel

$\mathrm{V}=$ Viertel

$\mathrm{V} \cdot=$ punktierte Viertel

$\mathrm{H}=$ Halbe

Es fällt auf, dass wir es bei den schnelleren Werten teilweise mit wesentlich schnelleren Tempi zu tun haben als heute üblich. Extrem erscheint die Angabe für das Lied "Der Neugierige". Die Quelle gibt Viertel = 88, im zweiten Teil („O Bächlein meiner Liebe") 80 Schläge pro Minute an. Das ist etwa das doppelte Tempo von Fischer-Dieskau und Moore. Clara Klatte merkt an, die Angabe habe sie "einigermassen überrascht". Zwar liegt die Vermutung nahe, dass ein Über-

7 Franz Schubert, Die schöne Müllerin, Dietrich Fischer-Dieskau, Gerald Moore, LP, Deutsche Grammophon 1972. 
tragungsfehler vorliegt und Stockhausen nicht Viertel, sondern Achtel meinte - zumal Schubert das Lied mit der Vortragsbezeichnung "Langsam" überschrieben hat. Andererseits gibt der Stockhausen-Schüler Johannes Messchaert in den 1920er Jahren immerhin Viertel $=60$ an: ein immer noch rasches Tempo, das auch Richard Tauber in dieser Zeit für das Lied nahm. „Der Neugierige" erhält so im Anfangsteil einen tänzerischen Charakter.

In unseren Ohren ungewöhnlich dürfte auch die Auffassung der Nr. 16 "Die liebe Farbe" klingen. Schubert schrieb „Etwas langsam” darüber. Unsere Quelle gibt Achtel = 104 an. Eine historische Schallplattenaufnahme von 1928 mit dem Tenor Hans Duhan und dem Pianisten Ferdinand Foll verwirklicht diese Tempovorstellung ungefähr. Sie kommen auf eine Spieldauer von 3 Minuten und 4 Sekunden. In "Die liebe Farbe" bringt die Tempoveränderung eine Verschiebung des emotionalen Ausdrucks mit sich: Schon die schnelle Frequenz des AchtelOstinatos im Klavier kann zornig wirken, während eine langsame Tonrepetition einen resignativklagenden Charakter darzustellen scheint.

Auch in anderen historischen Einspielungen dieses Liedes begegnen wir deutlich schnelleren Tempi als in modernen Einspielungen. Hier eine kleine Liste ausgewählter Aufnahmen mit Spieldauer (die aufgrund der Temposchwankungen leichter zu ermitteln ist als die durchschnittliche Metronomzahl):

1905 Emmy Destinn, unbekannter Pianist

1935 Gerhard Hüsch, Hanns-Udo Müller

1957 Walther Ludwig, Walther Bohle

1992 Christoph Pregardien, Andreas Staier

2002 Matthias Goerne, Eric Schneider

2013 Florian Boesch, Malcolm Martineau

2017 Christian Gerhaher, Gerold Huber ca. 2:32 Min. ${ }^{8}$

3:13 Min. ${ }^{9}$

3:00 Min. ${ }^{10}$

4:09 Min. ${ }^{11}$

5:06 Min. ${ }^{12}$

5:12 Min. ${ }^{13}$

4:39 Min. ${ }^{14}$

Einige moderne Interpreten geben dem Lied also doppelt so viel Zeit wie es Emmy Destinn vor über 100 Jahren tat. Gibt es im Laufe der letzten 100 Jahre Interpretationsgeschichte eine

8 „Emmy Destinn - Die liebe Farbe [Die schöne Müllerin, Op. 25, D.795] - 1905”, auf: Youtube, <https://www. youtube.com/watch?v=Lb1xwVqMurs> (29.08.2020). Die Spieldauer kann etwas länger gewesen sein, da die Umdrehungszahl der Schallplatten zu dieser Zeit nicht normiert war.

9 "Gerhard Hüsch ,Die liebe Farbe' Die schöne Müllerin", auf: Youtube, <https://www.youtube.com/ watch?v=tbzGvnE6ICM> (29.08.2020).

10 "Walther Ludwig; ,Die schöne Müllerin'; Franz Schubert”, auf: Youtube, <https://www.youtube.com/ watch?v=HODTLxOWtuA\&t=2823s> (29.08.2020).

11 „Die schöne Müllerin, Op. 25, D. 795: No. 16, Die liebe Farbe. ,In Grün will ich mich kleiden',auf: Youtube, <https:// www.youtube.com/watch?v=oYsiDM_ZRaM> (29.08.2020).

12 "Schubert: Die schöne Müllerin, D.795 - 16. Die liebe Farbe", auf: Youtube, <https://www.youtube.com/ watch?v=8XisOhmGaQ0> (29.08.2020).

13 „Florian Boesch; ,Die liebe Farbe'; Die schöne Müllerin; Franz Schubert”, auf: Youtube, <https://www.youtube. com/watch?v=cFBAc9hxajl> (29.08.2020).

14 „Die schöne Müllerin, Op. 25, D. 795: 16. Die liebe Farbe”, auf: Youtube, <https://www.youtube.com/ watch?v=9xeeG78FPW0 > (29.08.2020). 
Tendenz zur Verlangsamung? Tatsächlich lässt sich dies für alle vier bei Klatte/Stockhausen vergleichsweise rasch genommenen "Müllerlieder" (Nr. 4, 6, 16 und 20) beobachten, ebenso bei manchen anderen Schubertliedern. Verallgemeinern lässt sich diese Tendenz jedoch nicht. Das berühmte "An die Musik" sang die Mezzosopranistin Elena Gerhardt mit Artur Nikisch am Klavier im Jahre 1911 in getragenen vier Minuten (mit extremen Rubati); die Interpreten der letzten fünf Jahrzehnte führen es im Durchschnitt in zweieinhalb Minuten auf.

Im Fall der Stockhausen'schen Müllerlieder-Tempi kommen neben möglichen zeittypischen Tempoauffassungen auch individuelle bzw. kontextuelle Gründe in Betracht. Stockhausens Interpretation des Liedes "Die liebe Farbe" könnte aus dem Zusammenhang seiner Aufführungen zu verstehen sein. In einigen seiner Müllerin-Konzerte ergänzte Stockhausen den Zyklus durch den Vortrag der von Schubert nicht vertonten Gedichte Müllers. ${ }^{15}$ Eines davon, "Erster Schmerz, letzter Scherz" steht zwischen den vertonten Liedern "Eifersucht und Stolz" und "Die liebe Farbe".

In diesem Gedicht sitzt der Müllerbursche am Bach, bläst den Kindern, wie angekündigt, "schöne Tänz' und Lieder vor". Da sieht er hinter dem vielbesungenen Fenster die Müllerin in den Armen des Jägers, während der Bach lustig rauscht.

„ICh will mit Rosenblättern / Den Mühlensteg bestreun: / Der Steg hat mich getragen / Zu dir, Herzliebste mein! / Und wenn der stolze Jäger / Ein Blättchen mir zertritt, / Dann stürz, o Steg, zusammen/Und nimm den Grünen mit! /Und trag ihn auf dem Rücken / Ins Meer, mit gutem Wind, / Nach einer fernen Insel / Wo keine Mädchen sind. ${ }^{16}$

Ton und Inhalt dieses Gedichts sind also eher von Wut und Rachephantasie geprägt als von Resignation. Vor diesem Hintergrund erscheint es schlüssig, auch das folgende Lied, „Die liebe Farbe", eher sarkastisch bis aggressiv einzufärben als klagend und selbstmitleidig. Doch auch ohne den Gedicht-Einschub könnte das Tempo einer Logik folgen. Wenn Stockhausen für die fünf Lieder Nr.12 bis Nr. 16 in Folge fast identische Metronomzahlen vorsah, wollte er möglicherweise durch eine übergreifende metrische Einheit einen dramaturgischen Zusammenhang, eine "Klammer" herstellen.

Eduard Hanslick würdigte im Jahr 1860 den "Vortheil" von Stockhausens Gesamtaufführung des Liederzyklus, "das bisher lyrisch Vereinzelte auch einmal dramatisch auffassen zu können". Zugleich warnte der prominente Kritiker davor, diese Praxis zur Gewohnheit werden zu lassen, denn die "vollständige Abrollung" der "idyllischen Bildchen" könne "allmälig monoton" werden. ${ }^{17}$

15 Natasha Loges „Julius Stockhausen's Early Performances of Franz Schubert's Die Schöne Müllerin", in: 19thCentury Music 41, H. 3 (2018), S. 206-224.

16 Wilhelm Müller, Die Winterreise, hrsg. von Hans-Rüdiger Schwab, Frankfurt a.M. 1994, S. $30 f$.

17 Hanslick, Geschichte des Concertwesens, S. 214. 
Julius Stockhausen war freilich kein dramatischer Sänger. Sein Vortragsstil beim Liedgesang wurde von Zeitgenossen oft mit Begriffen wie "Natürlichkeit" oder "schmucklos-einfach"18 charakterisiert. Die Schriftstellerin und Sängerin Monika Hunnius berichtet in ihren Erinnerungen von ihrer ersten Gesangsstunde bei Stockhausen, in der der Meister sie mit den Worten konfrontiert: „Sie singen nicht einfach genug, Sie wollen zu viel. Sie wollen in jedes Wort einen Ausdruck pressen und überladen damit das Lied." ${ }^{19}$ Unter diesem Aspekt liegt ein weiterer Gedanke nahe: Diejenigen Müllerin-Lieder, für die Stockhausen wohl schnelle Tempi wählte, vor allem "Der Neugierige”, „Die liebe Farbe” und auch „Des Baches Wiegenlied” haben im weitesten Sinne volksliedhaften Charakter, repräsentieren also durch ihre Strophenform und/oder durch eine dem Volkslied nachempfundene Melodik ein Einfachheits- und Natürlichkeitsideal der Romantik. Stockhausen könnte danach gestrebt haben, gerade in diesen Liedern seine Vorstellung von Schlichtheit auch durch das Tempo zu verwirklichen, gleichsam Gewicht zu verringern - und zugleich der befürchteten "Monotonie" des ganzen Zyklus entgegenzuwirken. ${ }^{20}$

So sind verschiedene Gründe denkbar, warum Julius Stockhausen für einige "Müllerlieder" schnelle Tempi vorgeschlagen haben könnte. Die Tempobezeichnungen erscheinen, wie schon Clara Klatte bemerkte, teilweise überraschend, bei näherer Betrachtung in der Perspektive der historischen Interpretationsforschung jedoch nicht unglaubwürdig. Und angesichts mancher moderner Interpretationen der "Lieben Farbe" als eine mehr als fünf Minuten dauernde Schmerz-Introspektion wirkt Stockhausens Mahnung, nicht „in jedes Wort einen Ausdruck pressen" zu wollen, sogar bemerkenswert aktuell.

Zitation: Christian Lehmann, „Tempobezeichnungen von Julius Stockhausen für Die schöne Müllerin: Ein Quellenfund", in: Freie Beiträge zur Jahrestagung der Gesellschaft für Musikforschung 2019, hrsg. von Nina Jaeschke und Rebecca Grotjahn (= Musikwissenschaft: Aktuelle Perspektiven. Bericht über die Jahrestagung der Gesellschaft für Musikforschung 2019 in Paderborn und Detmold, Bd. 1), Detmold 2020, S. 191-200, DOI: 10.25366/2020.61.

18 Z. B. Recensionen und Mittheilungen über Theater und Musik, H. 18 (1860), S. 275; Schwäbischer Merkur, Nr. 39 (16. Februar 1869).

19 Monika Hunnius, Aus Heimat und Fremden, Heilbronn 1929, S. 50.

20 Für eine ausführliche Abhandlung der Schubert-Aufführungen durch Stockhausen sei auf Martin Günther, Kunstlied als Liedkunst, Stuttgart 2016, S. 238-279 verwiesen. 


\section{Abstract}

Julius Stockhausen (1826-1906) is known as the first singer who performed Schubert's Die schöne Müllerin as a whole cycle in a public concert. A celebrated lieder singer, friend of Brahms, author of pedagogical works, and most prominent German singing teacher of his time, Stockhausen's influence on lieder singing cannot be overestimated.

Recently a manuscript with "Tempo indications for Die schöne Müllerin by Julius Stockhausen" has been found. The document, containing tempo suggestions for every single lied of the song cycle, was written down by the German singing professor Clara Klatte (1867-1945). Some of the suggested tempi are surprisingly fast.

In this article the authenticity of the document is discussed under several aspects. The KlatteStockhausen document is published here for the first time.

\section{Kurzvita}

Christian Lehmann ist als Musikwissenschaftler, Sänger sowie Gesangspädagoge tätig und unterrichtet an den Universitäten München und Regensburg. Zu seinen Veröffentlichungen zählen das in mehrere Sprachen übersetzte Buch Der genetische Notenschlüssel, der Dokumentarfilm Wiegenlied und Schlachtgesang (BR/arte) und mehrere Artikel für das beim Laaber-Verlag erschienene Lexikon der Gesangsstimme. 


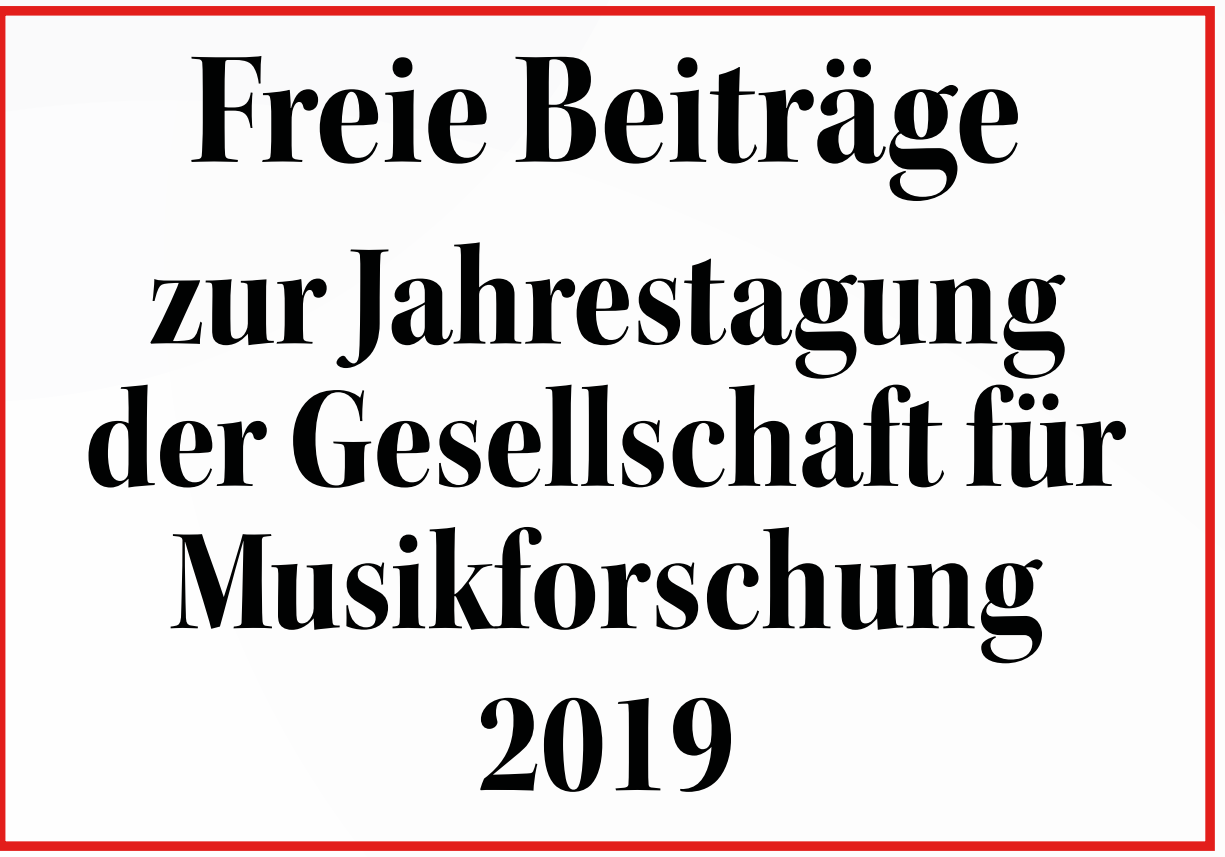

Herausgegeben von Nina Jaeschke und Rebecca Grotjahn

Musikwissenschaft: Aktuelle Perspektiven 1 
Freie Beiträge 


\section{Musikwissenschaft: Aktuelle Perspektiven}

Bericht über die Jahrestagung der Gesellschaft für Musikforschung 2019 in Paderborn und Detmold

Herausgegeben von Rebecca Grotjahn und Nina Jaeschke

Band 1 


\section{Freie Beiträge}

\section{zur Jahrestagung der Gesellschaft für Musikforschung 2019}

Herausgegeben von Nina Jaeschke und Rebecca Grotjahn

Detmold: Musikwissenschaftliches Seminar der Universität Paderborn und der Hochschule für Musik Detmold 2020 
DOI: $10.25366 / 2020.42$

Online-Version verfügbar unter der Lizenz: Urheberrecht 1.0, $<$ https://rightsstatements.org/page/InC/1.0/?language=de>

Bibliografische Information der Deutschen Nationalbibliothek

Die Deutsche Nationalbibliothek verzeichnet diese Publikation in der Deutschen Nationalbibliografie; detaillierte bibliografische Daten sind im Internet über http://dnb.d-nb.de abrufbar.

\section{Impressum}

Redaktion: Nina Jaeschke, Rebecca Grotjahn und Jonas Spieker Satz: Nina Jaeschke

(C) Musikwissenschaftliches Seminar der Universität Paderborn und der Hochschule für Musik Detmold 2020 


\section{INHALT}

Vorwort $\quad$ IX

Komponieren für das Radio: Akteure, Diskurse, Praktiken $\quad 1$

Musikwissenschaft - Feminismus - Kritik: Ein Generationenaustausch 6

\section{Stefan Alschner}

Der Wagner-Sänger Joseph Aloys Tichatschek - Vom Nachlass zum Netzwerk

\section{Alenka Barber-Kersovan}

Songs for the Goddess. Das popmusikalische Neo-Matriarchat zwischen Ethno-Beat,

erfundenen Traditionen und kommerzieller Vermarktung

Elias Berner, Julia Jaklin, Peter Provaznik, Matej Santi, Cornelia Szabó-Knotik

Musikgeschichte anders erzählen? Das Beispiel der 1970er in Österreich.

Musikhistoriographie in der Zeit der Digitalisierung

\section{Mauro Fosco Bertola}

„Ein Laut so klagevoll”. Lohengrin zwischen Richard Wagner und Salvatore Sciarrino

\section{Matthieu Cailliez}

Europäische Rezeption der Berliner Hofoper und Hofkapelle von 1842 bis 1849

\section{lacopo Cividini}

Zwischen klassischer Musikphilologie und angewandter Informatik:

Die Digitale Mozart-Edition (DME) der Stiftung Mozarteum Salzburg

\section{Marko Deisinger}

Fortschrittliche Technologie im Dienste eines Antimodernisten.

Heinrich Schenker und der österreichische Rundfunk

\section{Norbert Dubowy}

Vom Kritischen Bericht zur Kritischen Dokumentation am Beispiel der Digital-interaktiven Mozart-Edition

\section{Markus Engelhardt}

Musik zwischen Nation Building und Internationalität. Italien um 1900

\section{Maryam Haiawi}

Das Oratorium im Spannungsfeld der Konfessionen: 


\section{Judith I. Haug}

"Manch eine*r liegt, morgens noch trunken, im Rosengarten" - Rekonstruktionen

osmanischer Musikgeschichte in Gesangstextsammlungen

\section{Renate Koch}

Marcel Prawy und das erste Broadway-Musical im Österreich der Nachkriegszeit

Susanne Kogler, Julia Mair, Juliane Oberegger, Johanna Trummer

Erich Marckhl - Musikausbildung in der Steiermark nach 1945.

Brüche und Kontinuitäten

\section{Marie-Anne Kohl}

Die weinende Jury. "Geschlechtslose" Tränen bei globalen Musik-Castingshows?

\section{Fabian Kolb}

Tanztheater und filmische Ästhetik. Cineastische Einflüsse und Gestaltungsweisen in den Kompositionen für die Ballets Suédois 1920-1925

\section{Christian Lehmann}

Tempobezeichnungen von Julius Stockhausen für Die schöne Müllerin:

Ein Quellenfund

\section{Martin Link}

Signum et gens - Zur Gendersemiotik in Clara und Robert Schumanns Liederzyklus Liebesfrühling

\section{Livio Marcaletti}

„Strafspiel" und satirische Stilmittel in musikdramatischen Gattungen des frühen 18. Jahrhunderts

\section{Tobias Marx, Martin Lissner}

Thüringer Musikszene - Jugendmusikredaktionen als außerschulische musikbezogene Bildungskontexte

\section{Maho Naito}

Die Parallelität der Entstehungsprozesse der ersten beiden Symphonien Gustav

Mahlers: Instrumentation, Revision und Dirigierpraxis

\section{Elisa Novara}

Eine Schumann-Werkstatt? Zur Übertragbarkeit der Methoden vom Projekt 
Theodora Oancea, Joachim Pollmann, Jonas Spieker

Kollaborateure - Involvierte - Profiteure. Erarbeitung eines Online-Lexikons zur

Musik in der NS-Zeit

\section{Kiron Patka}

„Ich wollte eigentlich Sängerin werden." Berufsselbstbilder von Tontechniker*innen im Radio

\section{Siegwart Reichwald}

Die Leiden der jungen Clara: Das Klaviertrio Opus 17 als Ausdruck einer Neu-

Romantikerin

\section{Elisa Ringendahl}

Lied versus Oper - Pole musikalischer Gattungen bei Oscar Bie

\section{Benedikt Schubert}

Struktur und Exegese. Über Eigentümlichkeiten in der Arie "Des Vaters Stimme ließ sich hören" (BWV 7/4)

Uwe Seifert, Sebastian Klaßmann, Timo Varelmann, Nils Dahmen

Computational Thinking in der Musikwissenschaft: Jupyter Notebook als Umgebung

für Lehre und Forschung

\section{Yusuke Takamatsu}

Synthese als Modus der Prozessualität bei Schubert:

Sein spezifisches Wiederholungsprinzip im langsamen Satz

\section{Daniel Tiemeyer}

Johann Nepomuk Hummels Sonate in fis-Moll Op. 81 - Studien zu Entstehungs-

hintergrund, Rezeption und formalerStruktur

\section{Andrea van der Smissen}

Musikalische Innovation im Umfeld der Moderne und historischen Avantgarde in Ungarn

\section{Tim Ziemer, Holger Schultheis}

Psychoakustische Sonifikation zur Navigation in bildgeführter Chirurgie

\section{Magdalena Zorn}

Musik mit dem Radio hören: Über den Begriff der musikalischen Aufführung 
Gabriele Buschmeier in memoriam 


\section{Vorwort}

Die vorliegenden Bände dokumentieren die Jahrestagung der Gesellschaft für Musikforschung 2019. In den dreieinhalb Tagen vom 23. bis zum 26. September 2019 wurden in Paderborn und Detmold nicht weniger als 185 Beiträge präsentiert, verteilt auf diverse Symposien, Round tables, Freie Sektionen und Postersessions. Sie alle auf einen Nenner bringen zu wollen, ist ein Ding der Unmöglichkeit - und das ist gut so, ist es doch Ziel der Jahrestagungen, die große Vielfalt der Themen und Methoden des Faches Musikwissenschaft abzubilden. Um die thematische Vielfalt der freien Referate angemessen abbilden zu können und gleichzeitig den inhaltlichen Schwerpunkten der beiden hier publizierten Hauptsymposien ausreichend Raum bieten zu können, erscheinen diese in drei Bänden.

„Musikwissenschaft: Aktuelle Perspektiven": Der Titel der kleinen Reihe ist keine Verlegenheitslösung. Musikwissenschaft im Kontext der Digital Humanities; Musikwissenschaft und Feminismus; Musik und Medien; Musikalische Interpretation - schon die Felder, die von den vier Hauptsymposien bespielt wurden, wären noch vor wenigen Jahrzehnten allenfalls an der Peripherie das Faches zu finden gewesen. Sie entsprechen Arbeitsschwerpunkten der Lehrenden am Musikwissenschaftlichen Seminar der Universität Paderborn und der Hochschule für Musik Detmold, das die Tagung ausrichtete. Zugleich nehmen sie Bezug auf aktuelle Ereignisse und Entwicklungen. So erwuchs das von Andreas Münzmay und Joachim Veit organisierte Symposium „Brückenschläge - Informatik und Musikwissenschaft im Dialog" unmittelbar aus den Erfahrungen im Virtuellen Forschungsverbund Edirom (ViFE) und im fakultäten- und hochschulübergreifenden Zentrum Musik-Edition-Medien (ZenMEM). Der 200. Geburtstag von Clara Wieck/Schumann war der Anlass für das von Rebecca Grotjahn geleitete Symposium „Die Begleiterin - Clara Schumann, Lied und Liedinterpretation", das in enger Kooperation mit der Hochschule für Musik Detmold durchgeführt wurde. Das Hauptsymposium „Brückenschläge" wird in einem separaten Band publiziert (Bd. 3 der vorliegenden Reihe). Im Rahmen dieses Symposiums führte die von Stefanie Acquavella-Rauch geleitete Fachgruppe Digitale Musikwissenschaft eine Posterpräsentation durch, die von den Beiträger*innen erfreulicherweise zu kürzeren Texten umgearbeitet wurden, sodass sie hier ebenfalls, zusammen mit den Postern,

publiziert werden können. Hinzu kommen einige Beiträge, die bereits bei der Jahrestagung 2018 in Osnabrück präsentiert wurden. Auch das Hauptsymposium "Die Begleiterin" wird in einem eigenen Band (Bd. 2) publiziert. Die Beiträge zu den beiden anderen Hauptsymposien hingegen werden an anderen Orten veröffentlicht; in Band 1 („Freie Beiträge zur Jahrestagung der Gesellschaft für Musikforschung 2019") der vorliegenden Publikation finden sich jedoch Einführungen und Abstracts. Das Symposium „Komponieren für das Radio" unter Leitung von Antje Tumat und Camilla Bork (Katholieke Universiteit Leuven) behandelte Einflüsse des Mediums auf Kompositionsprozesse sowie durch radiophone Kompositionen bzw. radiophonen Klang ausgelöste Diskurse. Sarah Schauberger und Cornelia Bartsch (Universität Oldenburg) nahmen das 25-jährige Jubiläum der Fachgruppe Frauen- und Genderstudien zum Anlass für einen Generationenaustausch zum Thema "Musikwissenschaft - Feminismus - Kritik": Was wa- 
ren vor einem Vierteljahrhundert Inhalte und Aufgaben einer feministischen Musikwissenschaft und wie kann sich diese heute positionieren?

Bewusst haben wir im Tagungsbericht auf inhaltliche Eingriffe in die Beiträge verzichtet. ${ }^{1}$ Das gilt besonders für die Freien Referate: Es galt, den Charakter der Jahrestagung als Forum für ,freie', d. h. innovative und auch experimentelle Gedanken zu wahren. Einige Kolleg*innen, die die Tagung mit Vorträgen und Posterpräsentationen bereichert hatten, haben sich gegen eine Publikation im vorliegenden Band entschieden - sei es, weil sie eine Möglichkeit fanden, ihre Beiträge in einem inhaltlich passenderen Rahmen zu veröffentlichen, sei es, weil ihre Überlegungen in ihre entstehenden Qualifikationsschriften fließen sollen, oder sei es, weil sie von den Autor*innen in der vorgetragenen Form zunächst verworfen wurden. Auch damit erfüllt eine Freie-Referate-Sektion ihren Zweck: Die Diskussionen mit der versammelten Fach-Öffentlichkeit sollen dabei helfen, Gedanken weiterzuentwickeln und zu verändern. In diesem Sinne sei allen Beteiligten - den Autor*innen, den nichtpublizierenden Referent*innen und den Mit-Diskutant*innen - ganz herzlich gedankt für ihr Mitwirken bei der Tagung.

Unser herzlicher Dank gilt einer Reihe weiterer Personen, die zum Gelingen dieser drei Bände beigetragen haben. Hier ist besonders Jonas Spieker zu nennen, der uns tatkräftig bei der Redaktion geholfen hat. Andrea Hammes (SLUB Dresden) sei herzlich für die Aufnahme unseres Bandes auf musiconn.publish gedankt - wir freuen uns, damit unsererseits zur Etablierung dieser innovativen Publikationsplattform beizutragen.

Erneut möchten wir an dieser Stelle allen Menschen danken, die uns bei der Organisation, Ausrichtung und Finanzierung der Tagung selbst unterstützt haben: der Präsidentin der Universität Paderborn, Prof. Dr. Birgitt Riegraf, dem Rektor der Hochschule für Musik Detmold, Prof. Dr. Thomas Grosse, den Kolleginnen und Kollegen der beiden beteiligten Hochschulen, dem Vorstand der Gesellschaft für Musikforschung, der Universitätsgesellschaft Paderborn und allen Sponsoren. Besonders dankbar sind wir den Mitarbeiter*innen und den studentischen bzw. wissenschaftlichen Hilfskräften des Musikwissenschaftlichen Seminars, die bei der Vorbereitung und Ausrichtung der Tagung immensen Einsatz zeigten - stellvertretend sei an dieser Stelle Johanna Imm erwähnt, die zusammen mit Nina Jaeschke das Herz des Organisationsteams bildete.

Wir widmen diese Reihe Dr. Gabriele Buschmeier, dem langjährigen Vorstandsmitglied der Gesellschaft für Musikforschung, die kurz vor der Publikation dieses Bandes unerwartet verstarb.

Detmold, im September 2020

Rebecca Grotjahn und Nina Jaeschke

Zitation: Rebecca Grotjahn und Nina Jaeschke, „Vorwort”, in: Freie Beiträge zur Jahrestagung der Gesellschaft für Musikforschung 2019, hrsg. von Nina Jaeschke und Rebecca Grotjahn (= Musikwissenschaft: Aktuelle Perspektiven. Bericht über die Jahrestagung der Gesellschaft für Musikforschung 2019 in Paderborn und Detmold, Bd. 1), Detmold 2020, S. IX-X, DOI: 10.25366/2020.43.

1 Freigestellt war den Autor*innen auch, ob sie sich für eine gendersensible Sprache entscheiden bzw. welche Form des Genderns sie bevorzugen. 